\title{
Efecto de marcadores genéticos moleculares en genes inducibles por hipoxia de bovinos criollos y Brown Swiss criados en los Andes de Perú
}

\section{Effect of molecular genetic markers on hypoxia-inducible genes of Creole and Brown Swiss cattle raised in the Peruvian Andes}

\author{
Gonzalo W. Gonzales Aparicio ${ }^{1,3,4}$, Marcos Calderon Montes ${ }^{1}$, \\ Gustavo A. Gutierrez Reynoso', Federico Abel Ponce de León Bravo ${ }^{2}$
}

\section{Resumen}

Los genomas de 41 bovinos de cinco razas existentes en la base de datos del SRANCBI, fueron usados para identificar 17 polimorfismos de nucleótido simple (PNSs), los cuales generan mutaciones no sinónimas en 10 genes inducibles por hipoxia. De ese total, solo tres PNSs ubicados en las posiciones c. $1081 \mathrm{G}>$ C, c. $2089 \mathrm{G}>$ A y c. $2101 \mathrm{G}>\mathrm{A}$ de los exones 7 y 12 del gen EPAS1 tuvieron frecuencias alélicas diferentes entre bovinos cárnicos y lecheros $(\mathrm{p}<0.05)$. Basados en los resultados del análisis bioinformático de los genomas, se eligieron regiones exónicas de los genes EPAS1, NOS2, EPO y VEGFA, que fueron estudiadas en 60 muestras de ADN de bovinos criollos y Brown Swiss criados en la región altoandina (3213-4309 $\mathrm{msnm}$ ). Los bovinos fueron agrupados por su concentración de hemoglobina $(\mathrm{Hb})$ en Brown Swiss con $\mathrm{Hb}$ alta (BSHbA) $(\mathrm{n}=13)$, Brown Swiss con $\mathrm{Hb}$ baja $(\mathrm{BSHbB})(\mathrm{n}=11)$, criollos con $\mathrm{Hb}$ alta $(\mathrm{CRHbA})(\mathrm{n}=13)$ y criollos con Hb baja $(\mathrm{CRHbB})(\mathrm{n}=12)$; además se tuvo un grupo de Brown Swiss con mal de altura (BSMA)

\footnotetext{
${ }^{1}$ Universidad Nacional Agraria La Molina, Lima, Perú

${ }^{2}$ University of Minnesota, St. Paul, MN, United States

${ }^{3}$ Universidad Nacional de San Antonio Abad del Cusco, Perú

${ }^{4}$ E-mail: gonzalo.wlad@gmail.com
}

Recibido: 23 de agosto de 2020

Aceptado para publicación: 30 de junio de 2021

Publicado: 27 de octubre de 2021

CLos autores. Este artículo es publicado por la Rev Inv Vet Perú de la Facultad de Medicina Veterinaria, Universidad Nacional Mayor de San Marcos. Este es un artículo de acceso abierto, distribuido bajo los términos de la licencia Creative Commons Atribución 4.0 Internacional (CC BY 4.0) [https:// creativecommons.org/licenses/by/4.0/deed.es] que permite el uso, distribución y reproducción en cualquier medio, siempre que la obra original sea debidamente citada de su fuente original 
$(\mathrm{n}=11)$. Los amplicones generados fueron secuenciados con la metodología Sanger, identificándose 10 PNSs. No se encontraron asociaciones entre estos PNSs y los grupos considerados. Únicamente un PNS ubicado en la posición intrónica Cr.19: 19403681T>C del gen NOS2 tuvo frecuencia diferente entre bovinos criollos y Brown Swiss $(\mathrm{p}<0.05)$; además dicho PNS se encontró ligado a otros dos que están ubicados en el exón 12 del mismo gen.

Palabras clave: genoma, haplotipo, desequilibrio de ligamiento, frecuencia de alelo menor, mal de pecho

\section{Abstract}

The genomes of 41 bovines of five breeds, existing in the SRA-NCBI database, were used to identify 17 single nucleotide polymorphisms (SNPs) which generate nonsynonymous mutations in 10 hypoxia-inducible genes. Of this, only three SNPs located in positions c. $1081 \mathrm{G}>\mathrm{C}, \mathrm{c} .2089 \mathrm{G}>\mathrm{A}$ and c. $2101 \mathrm{G}>\mathrm{A}$ of exons 7 and 12 of the $E P A S 1$ gene had different allelic frequencies between beef and dairy cattle $(\mathrm{p}<0.05)$. Based on the results of the bioinformatic analysis of the genomes, exonic regions of the EPAS1, NOS2, $E P O$ and $V E G F A$ genes were chosen, which were studied in 60 DNA samples from Creole and Brown Swiss cattle raised in the high Andean region (3213-4309 $\mathrm{m}$ above sea level). The cattle were grouped by their haemoglobin concentration $(\mathrm{Hb})$ in Brown Swiss with high $\mathrm{Hb}(\mathrm{BSHb})(\mathrm{n}=13)$, Brown Swiss with low $\mathrm{Hb}(\mathrm{BSHbB})(\mathrm{n}=11)$, Creoles with high $\mathrm{Hb}(\mathrm{CRHbA})(\mathrm{n}=13)$ and Creoles with low $\mathrm{Hb}(\mathrm{CRHbB})(\mathrm{n}=12)$; in addition, there was a Brown Swiss group with brisket disease (BSMA) $(n=11)$. The generated amplicons were sequenced with the Sanger methodology, identifying 10 SNPs; however, no associations were found between these SNPs and the groups considered. Only one SNP located at the intronic position Cr.19: 19403681T $>$ C of the NOS2 gene had a different frequency between Creole and Brown Swiss cattle $(\mathrm{p}<0.05)$. In addition, that SNP was found linked to two others that are in exon 12 of the same gene.

Key words: genome, haplotype, linkage disequilibrium, minor allele frequency, brisket disease

\section{INTRODUCCIÓN}

El ganado bovino criado en tierras altas presenta respuestas fisiológicas que determinan su grado de aclimatación a dicho entorno; destacándose la hipertensión arterial pulmonar como el carácter más asociado a dicha aclimatación (Holt y Callan, 2007; Zeng, 2016). Esta característica está determinada por el incremento del tejido muscular de las arteriolas pulmonares, reduciendo su diámetro interno, a diferencia de otros bóvidos como los yaks (Durmowicz et al., 1993). La vasoconstricción de las arteriolas pulmonares ocasiona un incremento de la resistencia al flujo sanguíneo en la que también participa el grado de viscosidad de la sangre como resultado del elevado número de glóbulos rojos, teniendo un efecto en la hipertensión arterial (Vogel et al., 2003). Dicha condición es usual en bovinos criados en ambientes como los Andes, donde los bovinos presentan recuentos altos de glóbulos rojos (RGR), hematocrito (HCT) y hemoglobina $(\mathrm{Hb})$ (Claxton y Ortiz, 1996; Ocampo, 2004). Sin embargo, poblaciones de bovinos criados en las tierras altas de Etiopía presentan valores hematológicos 
y de presión arterial pulmonar (PAP) bajos, a pesar de estar expuestos al estímulo hipóxico crónico, de allí que se les considera como animales adaptados a ese ambiente (Wuletaw et al., 2011).

Para identificar las características genéticas que permiten la aclimatación y adaptación a la altura de especies animales, entre ellas el bovino, se han estudiado diversos genes, entre ellos el gen denominado proteína 1 para dominio endotelial $P A S$ (EPAS1) (Hara et al., 1999; Simonson et al., 2010, Newman et al., 2011; Wang et al., 2014). Este gen codifica el factor inducible por hipoxia tipo 2 alfa (HIF2 $\alpha$ ), subunidad alfa sensible al oxígeno, de un heterodímero resultante de la unión con la subunidad común beta o translocador nuclear del receptor de aril hidrocarburos (ARNT) codificado por el gen $A R N T$. El heterodímero es altamente expresado en células endoteliales vasculares y tiene participación en la sobrerregulación de diversos genes como respuesta al estímulo hipóxico; entre ellos, el factor de crecimiento endotelial vascular $(V E G F A)$ y la eritropoyetina (EPO) (Hara et al., 1999).

En el gen EPAS1 se han encontrado mutaciones o polimorfismos de nucleótido simple (PNSs) en las posiciones de c.G1816G $>$ A y c.1828G $>$ A del exón 12, ambos formando un mismo haplotipo y generando variación en la secuencia de los aminoácidos p.A606T y p.G610S pertenecientes al dominio de degradación dependiente de oxígeno ODDD (Newman et al., 2015; Heaton et al., 2016) y que están presentes en el 75\% de bovinos Angus con PAP mayor a $50 \mathrm{mmHg}$ (Newman et al., 2015). Además, se han encontrado otras mutaciones no sinónimas en los exones 7, 9, 12 y 13 que generan variaciones en las posiciones p.E270Q, p.P362L, p.A671G y p.L701F pertenecientes a los dominios PAS-B, e ID de la proteína (Heaton et al., 2016). En equinos se encontró una mutación en la posición p.R144C que es conservada en diferentes especies animales, la cual amplifica la respuesta transcripcional mediada por EPASI como respuesta a la hipoxia sobre genes de endotelina 1 ( $E D N 1)$, lactato deshidrogenasa A $(L D H A)$, supresor tumoral von HippelLindau (VHL) y EPO (Liu et al., 2019).

En cuanto al gen $E P O$, se ha encontrado una mutación con deleción en la posición c. 32 delG del exón 2 que ocasiona una modificación del marco abierto de lectura (ORF) produciendo ARNm de cadena más corta, el cual genera eritropoyetina de cadena corta en mayor concentración en ciertos individuos que padecen de eritrocitosis (Zmajkovic et al., 2018). Por otro lado, en poblaciones de patos adaptados a condiciones de vida a gran altitud se encontraron PNSs en genes como el óxido nítrico sintetasa NOS, EPAS1, y factor inducible por hipoxia egl-9 homologo 1 $(E G L N)$ destacándose la presencia de mutaciones no sinónimas en los exones 6 y 12 del gen EPAS1 y en el EGLN se encontró un PNS ubicado en el exón 2 que no generó una variación en el aminoácido (Graham y McCracken, 2018). Por su parte, el gen del factor inducible por hipoxia $H I F 1$ expresado en condiciones de hipoxia actúa como regulador maestro de la homeostasis de oxígeno bajo condiciones de hipoxia (Semenza, 2005), en un proceso de cascada el cual media en la expresión de algunos de los genes antes descritos como el EPO y VEGFA (Gilbert-Kawai et al., 2014) y de genes implicados en el transporte y metabolismo de la glucosa como el transportador de solutos familia 2 miembro 1 (SLC2A1) o GLUT-1 en diferentes tejidos (Hayashi et al., 2004; Shao et al., 2014).

En lo que respecta al análisis del ADN de varias especies, se cuenta con información genómica generada por diversos grupos de investigadores que guardan archivos en repositorios como el perteneciente al NCBI (Stothard et al., 2015; Das et al., 2015). La información es libre y además se dispone de herramientas bioinformáticas que permiten evaluar regiones del genoma a fin de identificar PNSs que puedan servir como marcadores asociados a determinadas características de interés. En ese sentido, el presente estudio tuvo como objetivo identificar marcado- 
res genéticos de tipo PNS en regiones exónicas de 10 genes inducibles por hipoxia usando los genomas de un grupo de 41 bovinos de varias razas bovinas cárnicas y lecheras. Asimismo, verificar la existencia de algunos de estos PNSs en regiones exónicas de cuatro genes previamente evaluados y su posible asociación basada en la variación de sus frecuencias alélicas respecto a grupos de bovinos Brown Swiss con mal de altura y de bovinos criollos y Brown Swiss criados en los Andes que expresaron valores extremos de $\mathrm{Hb}$, toda vez que estas poblaciones son consideradas como rústicas y adaptadas a la altura.

\section{Materiales Y Métodos}

En una primera etapa se identificaron los PNSs en regiones exónicas de los genes EPAS1, HIFla, EDN1, ARNT, HIF3a, VEGFA, EPO, NOS2, EGLN y SLC2A1, a fin de ubicar mutaciones no sinónimas en las secuencias de ADN. Para ello se empleó la información genómica de 41 bovinos Brown Swiss ( $\mathrm{n}=6$ ), Holstein (11), Jersey (8), Aberdeen Angus (9) y Simmental (7), existente en la base de datos Sequence Read Archive (SRA) del National Center for Biotechnology Information (NCBI, EEUU) (Cuadro 1). La información de los genomas fue descargada en el servidor de la Universidad Nacional Agraria La Molina (UNALM) para su análisis:

- La identificación de los PNSs se efectuó mediante el alineamiento de las secuencias obtenidas y el genoma de referencia ARS-UCD1.2 disponible en el NCBI(GCF_002263795.1), utilizando las herramientas bioinformáticas Alineador Burrows-Wheeler (BWA), Picard tools, SamTools y BcfTools;

- En la indexación con el genoma de referencia se emplearon las herramientas BWA y SamTools (Li et al., 2009; Li y Durbin, 2010);
- En la creación de la secuencia «diccionario», el ordenamiento, la marcación de duplicados, la indexación y la unión de archivos se empleó la herramienta Picard tools (Picard Toolkit, 2019);

- El alineamiento de lecturas se hizo con la herramienta BWA;

- La identificación y llamado de variantes se hizo con la herramienta BcfTools (BCF Tools, 2019).

Se obtuvo archivo de extensión. $v c f$ con peso de $19.8 \mathrm{~GB}$, del cual se extrajeron los PNSs ubicados en las coordenadas correspondientes a los genes antes referidos, generándose archivos FASTA con las variantes encontradas. Estos archivos fueron alineados con sus correspondientes archivos de ARNm y de proteínas codificadas existentes en la base de datos de NCBI (Códigos de referencias en el Cuadro 2), usando las herramientas Mega Blast (https://blast.ncbi.nlm.nih.gov/Blast.cgi) y Splign (https:// www.ncbi.nlm.nih.gov/sutils/splign/ splign.cgi). De esta manera se identificaron los PNSs que generaban variación en la codificación de los aminoácidos de la proteína resultante. Para este grupo de PNSs se calcularon las frecuencias del alelo menor (MAF en inglés), el equilibrio de Hardy-Weinberg, y el distanciamiento genético usando la prueba G (Sokal y Rohlf, 1995), existentes entre los dos grupos conformados por bovinos productores de carne y leche empleando el programa GENEPOP v. 4.7. (https://genepop.curtin.edu.au/). Por último, se efectuó un estudio de desequilibrio de ligamiento (LD, por la sigla en inglés) entre PNSs y la identificación de posibles haplotipos usando el algoritmo denominado columna sólida de LD del programa Haploview v.4.2 (Barret et al., 2005)

En la segunda parte del estudio se eligieron cuatro regiones exónicas de los genes EPAS1, EPO, VEGFA y NOS2 a partir de los resultados del análisis bioinformático previo. Para ello, se emplearon 60 muestras de ADN provenientes de bovinos criollos y Brown 
Cuadro 1. Códigos de referencia de los genomas bovinos empleados para el estudio bioinformático

\begin{tabular}{ccccc}
\hline Holstein & Aberdeen Angus & Simmental & Jersey & Brown Swiss \\
\hline SRR1262663H & SRR1262630A & SRR1262806S & SRR1262789J & ERR2561415B \\
SRR1262755H & SRR1262651A & SRR1262818S & SRR1262790J & ERR2561395B \\
SRR1262787H & SRR1293227A & SRR1262831S & SRR1262791J & ERR1766310B \\
SRR1262788H & SRR1262647A & SRR1262833S & SRR1262802J & ERR1746320B \\
SRR1262785H & SRR1262628A & SRR1262839S & SRR1262797J & ERR1746326B \\
SRR1262780H & SRR1262648A & SRR1262846S & SRR1262803J & ERR1747023B \\
SRR1262756H & SRR1262644A & SRR1262815S & SRR1262794J & \\
SRR1262664H & SRR1262659A & & SRR1262793J & \\
SRR1262539H & SRR1262633A & & & \\
SRR1262660H & & & & \\
SRR1262754H & & & & \\
\hline
\end{tabular}

Swiss criados en zonas altoandinas de las regiones Cusco, Pasco y Puno, Perú, en altitudes comprendidas entre 3213 y 4309 msnm. Estas muestras fueron seleccionadas dentro de un grupo de 323 bovinos de los que se analizaron sus características hematológicas en un estudio comparativo entre grupos raciales criados en condiciones altoandinas. Las muestras de ADN de los 60 individuos fueron distribuidas en cuatro grupos diferenciados por su concentración de $\mathrm{Hb}$ (alta: $15.25 \pm 0.81 \mathrm{~g} / \mathrm{dl}$; baja: $9.59 \pm 1.40 \mathrm{~g} / \mathrm{dl}$ ), dividiéndose en Brown Swiss con $\mathrm{Hb}$ alta (BSHbA) ( $\mathrm{n}=13)$, Brown Swiss con Hb baja (BSHbB) $(n=11)$, criollos con $\mathrm{Hb}$ alta (CRHbA) $(n=13)$ y criollos con Hb baja $(\mathrm{CRHbB})(\mathrm{n}=12)$; además de un quinto grupo formado por 11 bovinos Brown Swiss con mal de altura (BSMA) que tuvieron una concentración de $\mathrm{Hb}$ de $15.77 \pm 2.82 \mathrm{~g} / \mathrm{dl}$.

Exones seleccionados de los genes descritos anteriormente fueron amplificados mediante la reacción en cadena de polimerasa (PCR) usando los cebadores que se detallan en el Cuadro 3. Las muestras fueron preparadas de manera que se obtuvieran volúmenes de $50 \mu 1$ con una concentración mínima de $\mathrm{ADN}$ de $60 \mathrm{ng} / \mu \mathrm{l}$. Los amplicones fueron corridos en gel de agarosa al 1.5\% para observar la calidad de las bandas y tamaño del amplicón generado. Luego, las muestras fueron remitidas para su secuenciación mediante metodología Sanger. Los resultados obtenidos (archivos . bsf 1 ) fueron analizados con el programa BioEdit v. 7.2 .5 (Hall, 1999) y la herramienta Clustal Omega, mediante el alineamiento de las secuencias de los exones amplificados para cada gen dentro de cada grupo de animales. Los resultados de este análisis fueron empleados para determinar las frecuencias alélicas de los PNSs y diferencias poblacionales entre grupos raciales y entre grupos según la concentración de Hb. También se calcularon los correspondientes LDs y se identificaron los haplotipos tal y como fue descrito anteriormente.

\section{Resultados}

\section{Análisis Bioinformático de 41 Genomas Bovinos}

Se encontraron 79 PNSs en las regiones exónicas de los 10 genes evaluados. De estos, 17 PNSs no son sinónimos, ocasionando una variación en la secuencia de aminoácidos (Cuadro 4). 
Cuadro 2. Códigos de referencia de genes, ARNm y proteínas empleadas para el análisis bioinformático

\begin{tabular}{lccc}
\hline \multirow{2}{*}{ Gen } & \multicolumn{3}{c}{ Referencia de la secuencia - NCBI, USA } \\
\cline { 2 - 4 } & Genómico & ARNm & Proteína \\
\hline EPAS1 & NC_037338.1 & NM_174725.2 & NP_777150.1 \\
$E P O$ & NC_037352.1 & NM_173909.2 & NP_776334.1 \\
SLC2A1 & NC_037330.1 & NM_174602.2 & NP_777027.1 \\
HIF1a & NC_037337.1 & NM_174339.3 & NP_776764.2 \\
VEGFA & NC_037350.1 & NM_001316955.1 & NP_001303884.1 \\
NOS2 & NC_037346.1 & NM_001076799.1 & NP_001070267.1 \\
ARNT & NC_037330.1 & NM_173993.1 & NP_776418.1 \\
$E D N 1$ & NC_037350.1 & NM_181010.2 & NP_851353.1 \\
$H I F 3 a$ & NC_037345.1 & NM_001105342.1 & NP_001098812.1 \\
$E G L N 1$ & NC_037355.1 & XM_024986696.1 & XP_024842464.1 \\
\hline
\end{tabular}

Cuadro 3. Cebadores diseñados para PCR de muestras de ADN de bovinos Brown Swiss y criollos (Perú)

\begin{tabular}{|c|c|c|c|c|}
\hline Loci & Referencia & Cebador & $\begin{array}{l}\text { Longitud } \\
(\mathrm{pb})\end{array}$ & Localización \\
\hline L1 & $\begin{array}{l}\text { EPAS1 } \\
\text { Exon7 }\end{array}$ & $\begin{array}{l}\text { F: GGGGTGAGAGTTAGGGAACC } \\
\text { R: CTCAAGGCCAGATTCCACAG }\end{array}$ & 423 & $\begin{array}{c}\text { Cr11: } 28650676+ \\
28651098\end{array}$ \\
\hline L2 & $\begin{array}{c}V E G F A \\
\text { Segmento } 7\end{array}$ & $\begin{array}{l}\text { F: CGAGCCCGGAAGATTAGAG } \\
\text { R: TCCTCCACCAACGTCTCTTC }\end{array}$ & 668 & $\begin{array}{c}\text { Cr23: } 17296368+ \\
17297035\end{array}$ \\
\hline L3 & $\begin{array}{l}E P O \\
\text { exon5 }\end{array}$ & $\begin{array}{l}\text { F: AGAAGGGCCCATCCATTCTA } \\
\text { R: CAGTCCATCCTGTCCCTGTT }\end{array}$ & 622 & $\begin{array}{c}\mathrm{Cr} 25: 35873365- \\
35873986\end{array}$ \\
\hline L4 & $\begin{array}{l}N O S 2 \\
\text { exon12 }\end{array}$ & $\begin{array}{l}\text { F: AGAGACATGTGGCCCCTCTT } \\
\text { R: GGATTTGTCACAAGGCTGCT }\end{array}$ & 548 & $\begin{array}{l}\text { Cr19:19403459- } \\
19404006\end{array}$ \\
\hline
\end{tabular}

Al comparar las frecuencias alélicas entre los grupos de bovinos cárnicos y lecheros se observaron diferencias en tres PNSs del gen EPAS1 ubicados en c. $1081 \mathrm{G}>\mathrm{C}$ del exón $7(\mathrm{p}<0.05)$ y c. $2089 \mathrm{G}>\mathrm{A}(\mathrm{p}<0.05)$, c. $2101 \mathrm{G}>\mathrm{A}(\mathrm{p}<0.01)$, ambos en el exón 12 . El tercer PNS ubicado en la posición c. $1358 \mathrm{C}>\mathrm{T}$ del exón 9 no tuvo una frecuencia diferente entre bovinos cárnicos y leche- ros $(\mathrm{p}>0.05)$. Asimismo, el resto de PNSs no tuvo una frecuencia alélica diferente entre los grupos cárnicos y lecheros (Cuadro 5).

Luego de efectuar el análisis general de desequilibrio de ligamiento (LD) con las 41 muestras, se observó que los dos PNSs del gen EPAS1 ubicados en el exón 12 presentaron una puntuación de logaritmo de probabili- 
Cuadro 4. Polimorfismos de nucleótido simple (PNSs) identificados en 41 genomas bovinos que generan mutaciones no sinónimas en 10 genes inducibles por hipoxia

\begin{tabular}{|c|c|c|c|c|c|c|c|c|c|c|}
\hline \multirow{2}{*}{ N. ${ }^{o}$} & \multirow{2}{*}{ Gen } & \multirow{2}{*}{$\begin{array}{c}\text { Posición } \\
\text { genómica } \\
\text { (ARS-UCD1.2) }\end{array}$} & \multirow{2}{*}{ Exón } & \multirow{2}{*}{ ARNm } & \multicolumn{2}{|c|}{ PNS } & \multirow{2}{*}{$\begin{array}{c}\text { Codón } \\
\text { Ref }\end{array}$} & \multicolumn{3}{|c|}{ Aminoácido } \\
\hline & & & & & Ref & Alt & & Ref & Alt & Posición \\
\hline 1 & EPAS1 & Cr.11: 28809802 & 7 & c. 1081 & G & $\mathrm{C}$ & $\overline{\text { GAA }}$ & $\mathrm{E}(\mathrm{Glu})$ & $\mathrm{Q}(\mathrm{Gln})$ & p.270 \\
\hline 2 & EPAS1 & Cr.11: 28817869 & 9 & c. 1358 & $\mathrm{C}$ & $\mathrm{T}$ & $\mathrm{CCG}$ & P(Pro) & $\mathrm{L}(\mathrm{Leu})$ & p.362 \\
\hline 3 & EPAS1 & Cr.11: 28821484 & 12 & c. 2089 & G & $\mathrm{A}$ & GCC & A(Ala) & $\mathrm{T}(\mathrm{thr})$ & p.606 \\
\hline 4 & EPAS1 & Cr.11: 28821496 & 12 & c. 2101 & G & $\mathrm{A}$ & GGT & G(Gli) & S(Ser) & p. 610 \\
\hline 5 & $E P O$ & Cr.25: 35873867 & 5 & c. 457 & $\mathrm{C}$ & $\mathrm{T}$ & GCA & $\mathrm{A}(\mathrm{Ala})$ & $\mathrm{T}(\mathrm{thr})$ & p. 153 \\
\hline 6 & HIFla & Cr.10: 73857457 & 2 & c. 482 & G & $\mathrm{C}$ & CGT & $\mathrm{R}(\operatorname{Arg})$ & P(Pro) & p. 183 \\
\hline 7 & HIFla & Cr.10: 73875740 & 12 & c. 2064 & A & $\mathrm{G}$ & ATG & $\mathrm{M}(\mathrm{Met})$ & $\mathrm{V}(\mathrm{Val})$ & p.629 \\
\hline 8 & $V E G F A$ & Cr.23: 17296719 & 7 & c. 2262 & $\mathrm{C}$ & $\mathrm{T}$ & ACG & $\mathrm{T}(\mathrm{Thr})$ & $\mathrm{M}(\mathrm{Met})$ & ND \\
\hline 9 & $V E G F A$ & Cr.23: 17296773 & 7 & c. 2316 & G & A & CGA & $\mathrm{R}(\operatorname{Arg})$ & $\mathrm{Q}(\mathrm{Gln})$ & ND \\
\hline 10 & NOS2 & Cr.19: 19403710 & 12 & c. 1543 & A & $\mathrm{G}$ & TTC & $\mathrm{F}($ Fen $)$ & $\mathrm{L}(\mathrm{Leu})$ & p. 515 \\
\hline 11 & NOS2 & Cr.19: 19402899 & 13 & c. 1594 & $\mathrm{C}$ & $\mathrm{T}$ & GCC & A(Ala) & $\mathrm{T}(\mathrm{Thr})$ & p.532 \\
\hline 12 & NOS2 & Cr.19: 19400180 & 16 & c. 1961 & G & $\mathrm{A}$ & GCC & $\mathrm{A}(\mathrm{Ala})$ & $\mathrm{V}(\mathrm{Val})$ & p. 654 \\
\hline 13 & NOS2 & Cr.19: 19399220 & 17 & c. 2131 & G & A & CTC & L(Leu) & $\mathrm{F}(\mathrm{Fen})$ & p.711 \\
\hline 14 & $A R N T$ & Cr.3: 19935387 & 8 & c. 853 & G & $\mathrm{T}$ & GTG & $\mathrm{V}(\mathrm{Val})$ & L(Leu) & p. 285 \\
\hline 15 & $E D N 1$ & Cr.23: 44159580 & 4 & c. 782 & $\mathrm{C}$ & $\mathrm{T}$ & GGA & G(Gly) & $\mathrm{R}(\operatorname{Arg})$ & p. 164 \\
\hline 16 & EDN1 & Cr.23: 44161432 & 2 & c. 422 & G & $\mathrm{T}$ & $\mathrm{CCC}$ & P(Pro) & $\mathrm{T}(\mathrm{Thr})$ & p.44 \\
\hline 17 & $H I F 3 a$ & Cr.18: 53579258 & 10 & c. 1189 & $\mathrm{C}$ & $\mathrm{G}$ & CTG & $\mathrm{L}(\mathrm{Leu})$ & $\mathrm{V}(\mathrm{Val})$ & p.392 \\
\hline
\end{tabular}

En color rojo ubicación de PNS dentro de codón

ARNm: ARN mensajero; Ref: Referencia; Alt: Alterno; ND: no definido

dades (LOD en inglés) de 5.33 y r $^{2}$ de 0.70. Cabe señalar que cuando se compararon los grupos de bovinos cárnicos y lecheros de forma independiente, no se observó dicho ligamiento en estos últimos, mientras que en el grupo de bovinos cárnicos el ligamiento tuvo

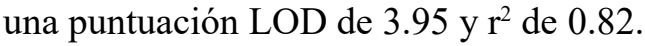

Por otro lado, con el análisis general de las 41 muestras, se observó que dos PNSs del gen NOS2 ubicados en las posiciones c.1961G >A (exon16) y c.1594C $>$ T (exón 13) tuvieron una puntuación LOD de 7.41 y un $\mathrm{r}^{2}$ de 0.61 , pero cuando se analizaron únicamente a bovinos cárnicos se observó un ligamiento entre los PNSs c.1594C > T (exón 13) y Cr.19:
19403681 T >C (intrón 12), los cuales tuvieron una puntuación LOD de 2.14 y r $^{2}$ de 0.52 ; mientras que en bovinos lecheros, se observó el ligamiento entre los PNSs c1594C $>\mathrm{T}$ (exón 13) y c.1961G $>$ A (exón 16) con una puntuación LOD de $5.9 \mathrm{y} \mathrm{r}^{2}$ de 0.75 , y estos a su vez estuvieron ligados con un tercer PNS ubicado en la posición Cr.19: 19403681T $>C$ (intrón 12), con puntuaciones LOD de $8.57 \mathrm{y}$ $6.68, \mathrm{y} \mathrm{r}^{2}$ de 0.92 y 0.83 , respectivamente.

Considerando las 41 muestras, se observaron otros PNSs que al parecer están ligados o son coheredados. Estos fueron el c. $2316 \mathrm{G}>\mathrm{A}$ ubicado en el exón $7 \mathrm{del}$ gen $V E G F A$ con el c.782C $>\mathrm{T}$ (exón 4) del gen 
Cuadro 5. Frecuencia del alelo menor (MAF) y equilibrio de Hardy-Weinberg (HW) de PNSs en genomas de bovinos

\begin{tabular}{|c|c|c|c|c|c|c|c|c|c|c|c|c|c|c|c|c|c|c|}
\hline \multirow{3}{*}{ లే } & \multirow{3}{*}{ 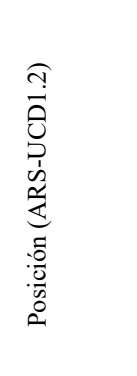 } & \multirow{3}{*}{ 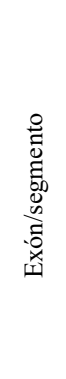 } & \multirow{3}{*}{$\frac{0}{\frac{0}{0}}$} & \multicolumn{8}{|c|}{ Bovinos lecheros } & \multicolumn{6}{|c|}{ Bovinos cárnicos } & \multirow{3}{*}{$\begin{array}{l}\overline{0} \\
0 \\
0 \\
0 \\
0 \\
0 \\
0\end{array}$} \\
\hline & & & & \multicolumn{2}{|c|}{ 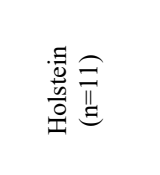 } & \multicolumn{2}{|c|}{ 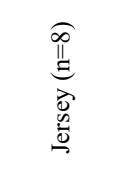 } & \multicolumn{2}{|c|}{ 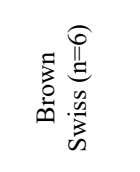 } & \multicolumn{2}{|c|}{ 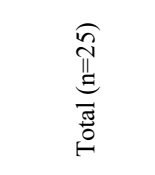 } & \multicolumn{2}{|c|}{ 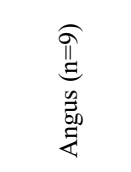 } & \multicolumn{2}{|c|}{ 岛 } & \multicolumn{2}{|c|}{ 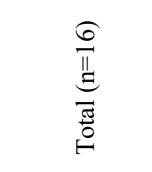 } & \\
\hline & & & & $\begin{array}{l}\overline{0} \\
2 \\
3\end{array}$ & $\sum_{\Sigma}^{L}$ & $\begin{array}{l}\text { 吾 } \\
2 \\
3\end{array}$ & $\sum_{\Sigma}^{L}$ & $\begin{array}{l}\bar{\pi} \\
2 \\
3 \\
3\end{array}$ & 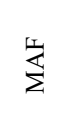 & $\begin{array}{l}\bar{a} \\
3 \\
3\end{array}$ & $\stackrel{5}{\Sigma}$ & $\begin{array}{l}\bar{E} \\
2 \\
3\end{array}$ & 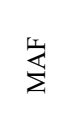 & $\begin{array}{l}\bar{m} \\
2 \\
3\end{array}$ & $\stackrel{L}{\Sigma}$ & $\begin{array}{l}\bar{E} \\
2 \\
3\end{array}$ & $\sum_{\Sigma}^{1}$ & \\
\hline$E P A S 1$ & $\begin{array}{c}\text { Cr.11: } \\
28809802\end{array}$ & 7 & $\mathrm{G}>\mathrm{C}$ & 0.48 & 0.32 & - & 0.06 & - & 0.00 & 1.00 & 0.160 & - & 0.00 & - & 0.00 & - & 0.000 & 0.021 \\
\hline EPAS1 & $\begin{array}{c}\text { Cr.11: } \\
28817869\end{array}$ & 9 & $\mathrm{C}>\mathrm{T}$ & 1.00 & 0.09 & - & 0.06 & - & 0.00 & 1.00 & 0.060 & - & 0.00 & 1.00 & 0.14 & 1.00 & 0.063 & 1.000 \\
\hline EPAS1 & $\begin{array}{c}\text { Cr.11: } \\
28821484\end{array}$ & 12 & $\mathrm{G}>\mathrm{A}$ & - & 0.05 & - & 0.00 & - & 0.00 & - & 0.020 & 0.46 & 0.33 & - & 0.00 & 1.00 & 0.188 & 0.038 \\
\hline EPASI & $\begin{array}{c}\text { Cr.11: } \\
28821496\end{array}$ & 12 & $\mathrm{G}>\mathrm{A}$ & - & 0.00 & - & 0.00 & - & 0.00 & - & 0.000 & 1.00 & 0.39 & - & 0.00 & 1.00 & 0.219 & 0.001 \\
\hline$E P O$ & $\begin{array}{c}\text { Cr.25: } \\
35873867\end{array}$ & 5 & $\mathrm{C}>\mathrm{T}$ & 1.00 & 0.14 & 0.21 & 0.19 & - & 0.08 & 0.38 & 0.140 & - & 0.06 & - & 0.07 & 1.00 & 0.063 & 0.316 \\
\hline HIFla & $\begin{array}{c}\text { Cr.10: } \\
73857457\end{array}$ & 2 & $\mathrm{G}>\mathrm{C}$ & - & 0.05 & - & 0.00 & - & 0.00 & - & 0.020 & - & 0.00 & - & 0.00 & - & 0.000 & 1.000 \\
\hline$H I F 1 a$ & $\begin{array}{c}\text { Cr.10: } \\
73875740\end{array}$ & 12 & $\mathrm{~A}>\mathrm{G}$ & - & 0.00 & - & 0.00 & - & 0.00 & - & 0.000 & - & 0.00 & - & 0.07 & - & 0.031 & 0.388 \\
\hline$V E G F A$ & $\begin{array}{c}\text { Cr.23: } \\
17296719\end{array}$ & 12 & $\mathrm{C}>\mathrm{T}$ & - & 0.05 & - & 0.00 & - & 0.08 & 1.00 & 0.004 & 1.00 & 0.28 & - & 0.00 & 0.30 & 0.156 & 0.105 \\
\hline$V E G F A$ & $\begin{array}{c}\text { Cr.23: } \\
17296773\end{array}$ & 7 & $\mathrm{G}>\mathrm{A}$ & 0.55 & 0.41 & - & 0.00 & - & 0.08 & 1.00 & 0.200 & - & 0.06 & - & 0.07 & 1.00 & 0.063 & 0.111 \\
\hline NOS2 & $\begin{array}{c}\text { Cr.19: } \\
19403710\end{array}$ & 12 & $\mathrm{~A}>\mathrm{G}$ & 0.51 & 0.27 & 1.00 & 0.25 & - & 0.08 & 0.30 & 0.220 & - & 0.00 & 0.44 & 0.29 & 0.20 & 0.125 & 0.390 \\
\hline NOS2 & $\begin{array}{c}\text { Cr.19: } \\
19402899\end{array}$ & 13 & $\mathrm{C}>\mathrm{T}$ & 0.26 & 0.45 & 1.00 & 0.13 & 1.00 & 0.41 & 1.00 & 0.380 & 0.49 & 0.39 & 1.00 & 0.43 & 1.00 & 0.406 & 0.817 \\
\hline NOS2 & $\begin{array}{c}\text { Cr.19: } \\
19400180\end{array}$ & 16 & $\mathrm{G}>\mathrm{A}$ & 0.09 & 0.50 & 1.00 & 0.13 & 1.00 & 0.42 & 0.41 & 0.360 & 0.49 & 0.39 & 1.00 & 0.36 & 0.59 & 0.375 & 1.000 \\
\hline NOS2 & $\begin{array}{c}\text { Cr.19: } \\
19399220\end{array}$ & 17 & $\mathrm{G}>\mathrm{A}$ & - & 0.00 & 0.20 & 0.19 & - & 0.00 & 0.06 & 0.060 & - & 0.00 & - & 0.00 & - & 0.000 & 0.276 \\
\hline$A R N T$ & $\begin{array}{c}\text { Cr.3: } \\
19935387\end{array}$ & 8 & $\mathrm{G}>\mathrm{T}$ & - & 0.00 & 1.00 & 0.13 & 1.00 & 0.25 & 1.00 & 0.100 & - & 0.00 & - & 0.00 & - & 0.000 & 0.085 \\
\hline$E D N 1$ & $\begin{array}{c}\text { Cr.23: } \\
44159580\end{array}$ & 4 & $\mathrm{C}>\mathrm{T}$ & 1.00 & 0.14 & - & 0.00 & - & 0.00 & 1.00 & 0.060 & - & 0.00 & - & 0.00 & - & 0.000 & 0.278 \\
\hline EDN1 & $\begin{array}{c}\text { Cr.23: } \\
44161432\end{array}$ & 2 & $\mathrm{G}>\mathrm{T}$ & 1.00 & 0.14 & - & 0.00 & - & 0.00 & 1.00 & 0.060 & 1.00 & 0.11 & - & 0.00 & 1.00 & 0.063 & 1.000 \\
\hline$H I F 3 a$ & $\begin{array}{c}\text { Cr.18: } \\
53579258\end{array}$ & 10 & $\mathrm{C}>\mathrm{G}$ & 1.00 & 0.41 & 1.00 & 0.19 & 0.39 & 0.42 & 1.00 & 0.380 & 1.00 & 0.11 & 1.00 & 0.36 & 0.27 & 0.344 & 0.815 \\
\hline NOS2 & $\begin{array}{c}\text { Cr.19: } \\
19403571\end{array}$ & I. 12 & $\mathrm{G}>\mathrm{A}$ & 1.00 & 0.14 & - & 0.06 & - & 0.00 & 1.00 & 0.080 & 1.00 & 0.33 & - & 0.00 & 0.44 & 0.188 & 0.180 \\
\hline NOS2 & $\begin{array}{c}\text { Cr.19: } \\
19403681\end{array}$ & I. 12 & $\mathrm{~T}>\mathrm{C}$ & 0.09 & 0.50 & 1.00 & 0.13 & 1.00 & 0.42 & 0.41 & 0.360 & 1.00 & 0.28 & 1.00 & 0.43 & 0.59 & 0.344 & 1.000 \\
\hline$E P O$ & $\begin{array}{c}\text { Cr.25: } \\
35873425\end{array}$ & 5 & $\mathrm{C}>\mathrm{T}$ & 1.00 & 0.18 & - & 0.00 & 1.00 & 0.08 & 1.00 & 0.120 & - & 0.06 & - & 0.00 & - & 0.031 & 0.239 \\
\hline$V E G F A$ & $\begin{array}{c}\text { Cr.23: } \\
17296897\end{array}$ & 7 & $\mathrm{G}>\mathrm{T}$ & 0.14 & 0.14 & 0.39 & 0.25 & - & 0.08 & 0.08 & 0.160 & 1.00 & 0.11 & 1.00 & 0.14 & 1.00 & 0.125 & 0.759 \\
\hline$S L C 2 A 1$ & $\begin{array}{c}\text { Cr.3: } \\
103320850\end{array}$ & 2 & $\mathrm{C}>\mathrm{T}$ & 1.00 & 0.32 & - & 0.06 & - & 0.00 & 0.49 & 0.160 & 0.34 & 0.22 & - & 0.00 & 0.19 & 0.125 & 0.759 \\
\hline $\mathrm{Co}$ & njunto & & & 0.91 & & 0.97 & & 0.99 & & 0.98 & & 0.99 & & 1.00 & & 0.98 & & 0.038 \\
\hline
\end{tabular}

I.12: Intrón 12 
Cuadro 6. Frecuencia del alelo menor (MAF)y equilibrio de Hardy-Weinberg (HW) de polimorfismos de nucleótido simple (PNSs) no sinónimos observados en bovinos criados en los Andes peruanos

\begin{tabular}{|c|c|c|c|c|c|c|c|c|c|c|c|c|c|}
\hline \multirow{3}{*}{ छี } & \multirow{3}{*}{ 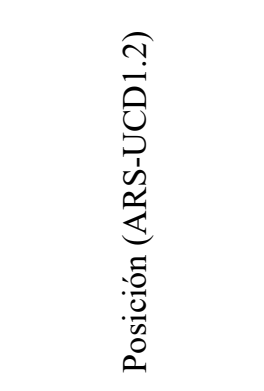 } & \multirow{3}{*}{ 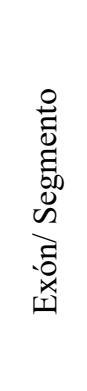 } & \multirow{3}{*}{$\frac{\frac{0}{0}}{\frac{0}{4}}$} & \multicolumn{5}{|c|}{ Concentración media de $\mathrm{Hb}$} & \multicolumn{5}{|c|}{ Tipo de bovinos } \\
\hline & & & & \multicolumn{2}{|c|}{ 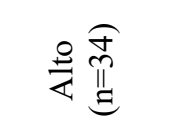 } & \multicolumn{2}{|c|}{ 产 } & \multirow{2}{*}{$\begin{array}{l}\vec{\pi} \\
\stackrel{7}{2} \\
2 \\
\stackrel{\overrightarrow{0}}{0} \\
0\end{array}$} & \multicolumn{2}{|c|}{ 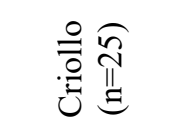 } & \multicolumn{2}{|c|}{ 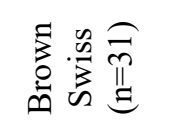 } & \multirow{2}{*}{$\begin{array}{l}\overrightarrow{⿱ 乛} \\
\stackrel{0}{0} \\
\overrightarrow{0} \\
\stackrel{0}{0} \\
0\end{array}$} \\
\hline & & & & $\begin{array}{l}3 \\
2 \\
2 \\
3\end{array}$ & $\sum^{2}$ & $\begin{array}{l}\overline{3} \\
2 \\
0 \\
3\end{array}$ & $\sum^{L}$ & & $\begin{array}{l}\overline{3} \\
0 \\
0 \\
3\end{array}$ & $\sum^{2}$ & $\begin{array}{l}\overline{3} \\
2 \\
3 \\
3\end{array}$ & $\sum^{L}$ & \\
\hline EPAS1 & Cr.11: 28809802 & 7 & $\mathrm{G}>\mathrm{C}$ & 1.00 & 0.041 & 0.13 & 0.087 & 0.427 & 0.20 & 0.100 & 1.00 & 0.029 & 0.124 \\
\hline NOS2 & Cr.19: 19403571 & I. 12 & $\mathrm{G}>\mathrm{A}$ & 1.00 & 0.136 & 1.00 & 0.136 & 1.000 & 1.00 & 0.140 & 1.00 & 0.133 & 1.000 \\
\hline NOS2 & Cr.19: 19403681 & I. 12 & $\mathrm{~T}>\mathrm{C}$ & 0.56 & 0.182 & 0.54 & 0.227 & 0.630 & 1.00 & 0.100 & 0.62 & 0.283 & 0.017 \\
\hline NOS2 & Cr.19: 19403710 & 12 & $\mathrm{~A}>\mathrm{G}$ & 1.00 & 0.061 & 1.00 & 0.068 & 1.000 & 1.00 & 0.060 & 1.00 & 0.067 & 1.000 \\
\hline NOS2 & Cr.19: 19403717 & 12 & $\mathrm{C}>\mathrm{T}$ & 0.55 & 0.152 & 0.54 & 0.182 & 0.794 & 0.55 & 0.220 & 1.00 & 0.117 & 0.197 \\
\hline$E P O$ & Cr.25: 35873425 & 5 & $\mathrm{C}>\mathrm{T}$ & 1.00 & 0.203 & 1.00 & 0.150 & 0.619 & 1.00 & 0.208 & 0.56 & 0.167 & 0.632 \\
\hline$E P O$ & Cr.25: 35873867 & 5 & $\mathrm{C}>\mathrm{T}$ & 1.00 & 0.081 & -- & 0.025 & 0.278 & 1.00 & 0.083 & 1.00 & 0.046 & 0.452 \\
\hline$V E G F A$ & Cr.23: 17296719 & 7 & $\mathrm{C}>\mathrm{T}$ & 1.00 & 0.106 & 1.00 & 0.068 & 0.528 & 1.00 & 0.063 & 1.00 & 0.113 & 0.509 \\
\hline$V E G F A$ & Cr.23: 17296773 & 7 & $\mathrm{G}>\mathrm{A}$ & 1.00 & 0.121 & 1.00 & 0.136 & 1.000 & 1.00 & 0.146 & 1.00 & 0.113 & 0.577 \\
\hline \multirow[t]{2}{*}{$V E G F A$} & Cr.23: 17296897 & 7 & $\mathrm{G}>\mathrm{T}$ & 0.22 & 0.091 & 0.65 & 0.136 & 0.541 & 1.00 & 0.063 & 0.09 & 0.145 & 0.228 \\
\hline & Conjunto & & & 0.99 & & 0.96 & & 0.993 & 0.99 & & 0.93 & & 0.409 \\
\hline
\end{tabular}

I.12: Intrón 12

Hemoglobina alta $(15.25 \pm 0.81 \mathrm{~g} / \mathrm{dl})$; baja: $(9.59 \pm 1.40 \mathrm{~g} / \mathrm{dl})$

$E D N 1$ y entre los PNSs c. $1081 \mathrm{G}>\mathrm{C}$ del gen EPAS1 con c. $2316 \mathrm{G}>\mathrm{A}$ del gen $V E G F A$. (LOD: 2.06). Sin embargo, en el caso específico de bovinos lecheros se observó una puntuación LOD de 2.04 entre c. $2316 \mathrm{G}>\mathrm{A}$ del gen $V E G F A$ y el c.233C $>\mathrm{T}$ del gen $S L C 2 A 1$

En lo que se refieren a los haplotipos del gen EPAS1, en el grupo de bovinos cárnicos se observó un bloque de cinco haplotipos formados por el PNS del exón $9 \mathrm{y}$ los dos reportados en el exón 12. Estos fueron CGG (71.9\%), CAA (18.8\%), TGG $(6.3 \%)$ y CGA (3.1\%). En el caso de los bovinos lecheros se obtuvo un grupo de cinco haplotipos formados por los PNSs de los exones 7, 9, y uno del exón 12 (c.2089G $>$ A); de ellos, los haplotipos GCG (79.2\%), CCG (12.8\%), GTG, (4.8\%) tuvieron una mayor frecuencia.

En el gen NOS2 en bovinos lecheros se observó un bloque conformado por siete haplotipos con los PNSs c. $2131 \mathrm{G}>\mathrm{A}$, c. $1961 \mathrm{G}>$ A, c. $1594 \mathrm{C}>$ T, Cr.19: 19403571G $>$ A y Cr.19: 19403681T >C. Los haplotipos más frecuentes fueron GGCGT (53.9\%), GATGC (28.0\%), AGCGT (6.0\%) y GATAC (5.9\%). Para el caso de los bovinos cárnicos se observó un bloque de siete haplotipos formados por los PNSs c.1594C $>\mathrm{T}$, Cr.19: 19403571G $>$ A, Cr.19: 19403681T $>C$ y c. $1543 \mathrm{~A}>\mathrm{G}$, siendo los más frecuentes CGTA (44.3\%), TGCA (21.2\%), CGTG $(11.8 \%)$. 
Cuadro 7. Frecuencia del alelo menor (MAF) y equilibrio de Hardy-Weinberg (HW) de polimorfismos de nucleótido simple (PNSs) observados en bovinos por raza y nivel de hemoglobina dentro de raza

\begin{tabular}{|c|c|c|c|c|c|c|c|c|c|c|c|c|c|c|}
\hline \multirow{2}{*}{ Ðี } & \multirow{2}{*}{ 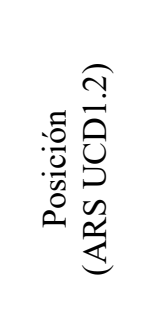 } & \multirow{2}{*}{ 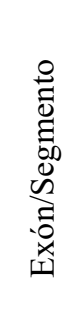 } & \multirow{2}{*}{$\frac{\frac{0}{0}}{\frac{1}{4}}$} & \multicolumn{2}{|c|}{ 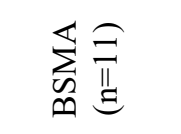 } & \multicolumn{2}{|c|}{ 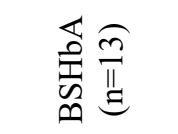 } & \multicolumn{2}{|c|}{ 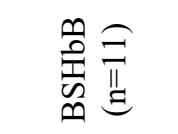 } & \multicolumn{2}{|c|}{ 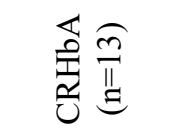 } & \multicolumn{2}{|c|}{ 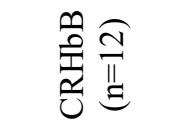 } & \multirow{2}{*}{ 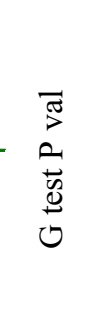 } \\
\hline & & & & $\begin{array}{l}3 \\
2 \\
2 \\
3\end{array}$ & 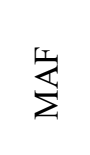 & $\begin{array}{l}\pi \\
i \\
2 \\
3 \\
1\end{array}$ & 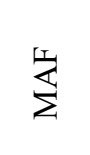 & $\begin{array}{l}\bar{\pi} \\
2 \\
2 \\
3\end{array}$ & $\sum_{\Sigma}$ & $\begin{array}{l}\pi \\
2 \\
2 \\
3\end{array}$ & $\sum_{\Sigma}$ & $\begin{array}{l}\pi \\
2 \\
2 \\
2\end{array}$ & $\stackrel{1}{\Sigma}$ & \\
\hline EPASI & $\begin{array}{c}\text { Cr.11: } \\
28809802\end{array}$ & 7 & $\mathrm{G}>\mathrm{C}$ & -- & 0.046 & -- & 0.039 & -- & 0.000 & -- & 0.039 & 0.25 & 0.167 & 0.255 \\
\hline NOS2 & $\begin{array}{c}\text { Cr.19: } \\
19403571\end{array}$ & I. 12 & $\mathrm{G}>\mathrm{A}$ & 1.00 & 0.278 & -- & 0.046 & 1.00 & 0.100 & 1.00 & 0.115 & 1.00 & 0.167 & 0.334 \\
\hline NOS2 & $\begin{array}{c}\text { Cr.19: } \\
\text { 19403681 }\end{array}$ & I. 12 & $\mathrm{~T}>\mathrm{C}$ & 0.46 & 0.333 & 1.00 & 0.182 & 0.22 & 0.350 & 1.00 & 0.077 & 1.00 & 0.125 & 0.103 \\
\hline NOS2 & $\begin{array}{c}\text { Cr.19: } \\
19403710\end{array}$ & 12 & $\mathrm{~A}>\mathrm{G}$ & -- & 0.000 & 1.00 & 0.091 & 1.00 & 0.100 & 1.00 & 0.077 & -- & 0.042 & 0.737 \\
\hline NOS2 & $\begin{array}{c}\text { Cr.19: } \\
19403717\end{array}$ & 12 & $\mathrm{C}>\mathrm{T}$ & -- & 0.056 & 1.00 & 0.182 & 1.00 & 0.100 & 0.37 & 0.192 & 1.00 & 0.250 & 0.447 \\
\hline$E P O$ & $\begin{array}{c}\text { Cr.25: } \\
35873425\end{array}$ & 5 & $\mathrm{C}>\mathrm{T}$ & 1.00 & 0.091 & 1.00 & 0.231 & 1.00 & 0.167 & 1.00 & 0.269 & 1.00 & 0.136 & 0.525 \\
\hline$E P O$ & $\begin{array}{c}\text { Cr.25: } \\
35873867\end{array}$ & 5 & $\mathrm{C}>\mathrm{T}$ & -- & 0.000 & 1.00 & 0.077 & -- & 0.056 & 1.00 & 0.154 & -- & 0.000 & 0.117 \\
\hline$V E G F A$ & $\begin{array}{c}\text { Cr.23: } \\
17296719\end{array}$ & 7 & $\mathrm{C}>\mathrm{T}$ & 1.00 & 0.150 & 1.00 & 0.091 & 1.00 & 0.100 & 1.00 & 0.083 & -- & 0.042 & 0.835 \\
\hline$V E G F A$ & $\begin{array}{c}\text { Cr.23: } \\
17296773\end{array}$ & 7 & $\mathrm{G}>\mathrm{A}$ & 1.00 & 0.100 & 1.00 & 0.091 & 1.00 & 0.150 & 1.00 & 0.167 & 1.00 & 0.125 & 0.946 \\
\hline$V E G F A$ & $\begin{array}{c}\text { Cr.23: } \\
17296897\end{array}$ & 7 & $\mathrm{G}>\mathrm{T}$ & -- & 0.050 & -- & 0.182 & -- & 0.200 & -- & 0.042 & -- & 0.083 & 0.365 \\
\hline Cor & njunto & & & 0.999 & & 1.000 & & 0.993 & & 1.000 & & 0.999 & & 0.255 \\
\hline
\end{tabular}

I.12: Intrón 12

Hemoglobina $(\mathrm{Hb})$ alta $(15.25 \pm 0.81 \mathrm{~g} / \mathrm{dl})$; baja: $(9.59 \pm 1.40 \mathrm{~g} / \mathrm{dl})$

BSMA: Brown Swiss con mal de altura, BSHbA: Brown Swiss con $\mathrm{Hb}$ alta, BSHbB: Brown Swiss con $\mathrm{Hb}$ baja, CRHbA: Criollo con $\mathrm{Hb}$ alta, $\mathrm{CRHbB}$ : Criollo con $\mathrm{Hb}$ baja

\section{PNSs en ADN de Bovinos Criollos y Brown Swiss}

Al secuenciar las 60 muestras de bovinos locales, se identificaron 10 PNSs en los amplicones obtenidos de los cuatro genes considerados. Estos guardan correspondencia con las posiciones identificadas en los 41 genomas presentados en los cuadros 4 y 5 .
Al comparar las frecuencias alélicas de estos PNSs entre bovinos criollos y Brown Swiss, solo fueron diferentes para dos PNSs ubicados en las posiciones Cr.19: 19403681T >C (intrón 12) y c.1536C $>$ T (exón 12) del gen NOS2. Este último PNS no genera una variación en la secuencia de aminoácidos p.E512E del producto. También se pudo observar que la frecuencia del PNS 
c. $1081 \mathrm{G}>\mathrm{C}$ del gen $E P A S 1$ fue de 0.10 en los bovinos criollos y en los bovinos CRHbB llegó a 0.17 (Cuadro 7). Además, cuando se agruparon las muestras en los dos grupos generales diferenciados por la concentración de $\mathrm{Hb}$ no se pudo apreciar diferencias en las frecuencias alélicas que pudieran estar asociadas con dichos grupos ( $\mathrm{p}>0.05)$ (Cuadro 6). Tampoco se apreciaron diferencias en las frecuencias de alelos entre los bovinos con mal de altura y los otros cuatro grupos de bovinos criollos y Brown Swiss (Cuadro 7).

En lo referido al análisis de desequilibrio de ligamiento de los PNSs, en el grupo Brown Swiss se observó la existencia de ligamiento entre los dos PNSs del gen NOS2 ubicados en las posiciones c. $1543 \mathrm{~A}>\mathrm{G}$ y c. $1536 \mathrm{C}>\mathrm{T}$, ambas dentro del exón 12 , con una puntuación LOD de 3.1 y un $\mathrm{r}^{2} 0.54$. En el grupo $\mathrm{BSHbB}$, el ligamiento tuvo una puntuación LOD de 2.2 y un $\mathrm{r}^{2}$ de 1.0. Además, en este grupo racial se identificó un bloque formado por cuatro haplotipos constituidos por los PNSs Cr.19: $19403681 \mathrm{~T}>\mathrm{C}$, c. $1543 \mathrm{~A}>\mathrm{G}$ y c. $1536 \mathrm{C}>$ T del gen NOS2: TAC, CAC, TGT y TAT existentes en el 61, 28, 6 y 5\% del grupo; mientras que en los bovinos criollos se observó un bloque conformado por los dos últimos PNSs del gen NOS2 antes referidos, encontrándose tres haplotipos: AC, AT, GT en el 78,16 y 6\% de los animales de este grupo.

Otro análisis para identificar haplotipos considerando esta vez a los 10 PNSs de los cuatro genes estudiados, permitió identificar un haplotipo común a los cinco grupos de estudio. Este fue CGGGTACGCC constituido por los PNSs VEGFA c. $2262 \mathrm{C}>\mathrm{T}, V E G F A$ c.2316G >A, VEGFA Cr.23: $17296897 \mathrm{G}>\mathrm{T}$, NOS2 Cr.19: 19403571G>A, NOS2 Cr.19: $19403681 \mathrm{~T}>\mathrm{C}, N O S 2$ c. $1543 \mathrm{~A}>\mathrm{G}, N O S 2$ c. $1536 \mathrm{C}>\mathrm{T}, E P A S 1$ c. $1081 \mathrm{G}>\mathrm{C}, E P O \mathrm{Cr} .25$ : $35873425 \mathrm{C}>\mathrm{T}$ y $E P O$ c. $457 \mathrm{C}>\mathrm{T}$. Sin embargo, la frecuencia de este varió entre grupos, observándose en bovinos BSMA y BSHbA una frecuencia de 0.51 y 0.55 , mientras que en bovinos BSHbB, CRHbA y CRHbB fue de $0.35,0.35$ y 0.33 , respectivamente. Tam- bién se identificaron dos haplotipos propios de bovinos criollos, el primero CGGGTATGCC con frecuencias de 0.15 en CRHbA y 0.13 en CRHbB y el segundo CGGGTACGTC con frecuencias de 0.13 en CRHbA y 0.08 en CRHbB. No se identificó un haplotipo que fuera único para el grupo de bovinos BSMA que tuviera una frecuencia alta como para ser considerado que este asociado con dicho grupo de animales.

\section{Discusión}

La elección de exones para amplificar en los grupos locales de bovinos criollos y Brown Swiss se basó en la identificación de PNSs no sinónimos existentes en los 41 genomas bovinos de la base datos SRA del NCBI. Para el caso del gen EPAS1 los resultados fueron concordantes en lo que se refiere a PNSs del exón 12 con los resultados de Newman et al. (2015) y Heaton et al. (2016); además, se evidenció la ausencia de PNSs en las posiciones c. $2089 \mathrm{G}>\mathrm{A}$ y c. $2101 \mathrm{G}>\mathrm{A}$ en bovinos lecheros, por lo que no se incluyó dicho exón en el análisis de las muestras de criollos y Brown Swiss locales; sin embargo, esto no implica que no existan dichas variantes en bovinos criollos, quedando pendiente dicha evaluación.

La variante del gen EPAS1 ubicada en la posición c. $1081 \mathrm{G}>\mathrm{C}$ del exón 7 que genera un cambio en p.E270Q, está presente en los genomas de Holstein y Jersey y aunque no fue identificada en Brown Swiss (Cuadro 5), se decidió analizarla. Ello permitió identificar el PNS en bovinos Brown Swiss y los criollos, aunque en baja frecuencia, no pudiéndose encontrar diferencias entre estos grupos raciales ni en cuánto a la concentración de $\mathrm{Hb}$, posiblemente debido al escaso número de muestras por grupo.

Un PNS que tuvo frecuencia alélica diferente entre los criollos y los Brown Swiss fue el Cr.19: 19403681T $>$ C del gen NOS2 $(p<0.05)$. Este PNS se ubica en una región 
intrónica, pero de acuerdo con los resultados de este estudio, está ligado con PNSs identificados en regiones exónicas del mismo gen. Como se sabe, el gen NOS2 está más vinculado con la repuesta inflamatoria (Accinelli y Lopez, 2018) y su trascripción está regulada por el incremento de las concentraciones del factor de necrosis tumoral alfa $(\mathrm{TNF} \alpha) \mathrm{e}$ interleucina beta (IL- $\beta$ ) en células endoteliales vasculares (Zulueta et al., 2002). Además, el $N O$ tiene efecto sobre el desarrollo de tejido muscular y endotelial vascular, participando en la regulación del tono vascular, entre otras funciones (Shao et al., 2015).

Debido a la importancia de estas funciones y considerando los procesos inflamatorios relacionados con el síndrome de mal de altura, se esperaba encontrar alguna asociación entre los PNSs del gen NOS2 con el grupo de bovinos BSMA que los diferenciara de los cuatro grupos de bovinos criollos y Brown Swiss diferenciados por su concentración de $\mathrm{Hb}$. Sin embargo, al no detectarse este tipo de asociación, se tendría que analizar las posibles diferencias en la expresión génica de genes inducibles por hipoxia entre los grupos considerados. Esta es una alternativa de trabajo con relación a estudios de adaptación a condiciones de altura (Verma et al., 2018), que podría permitir entender que los potenciadores o inhibidores del gen NOS 2 que controlan su expresión, así como la de muchos otros genes que participan en este proceso.

Por otro lado, la evaluación de haplotipos considerando a los 10 PNSs permitió identificar un haplotipo propio de los bovinos BSMA que pudiera tener una alta frecuencia en dicho grupo y aunque se pudo identificar dos haplotipos propios para bovinos criollos, la suma de ambas frecuencias observadas en CRHbA y CRHbB, estuvo en un rango entre 21 y $28 \%$, por lo que no son referenciales para esta población.
Con base a estos resultados, sería conveniente desarrollar un estudio de GWAS con mayor número de muestras, como los realizados por Wei et al. (2016) en ovinos o Liu et al. (2019) en equinos, e incluir información fenotípica referida a características fisiológicas que se presentan en el síndrome de mal de altura o que sean propias de animales adaptados a entornos de altitud, tal como se hacen en estudios para características de interés productivo o de adaptación ambiental (Martinez et al., 2014; De Leon et al., 2019), a fin de identificar PNSs de efecto mayor o que puedan estar implicados en los procesos metabólicos intermedios del síndrome de mal de altura y en la adaptación de poblaciones bovinas locales a la altura.

\section{Conclusiones}

- Se identificaron 17 polimorfismos de nucleótido simple (PNSs) en regiones exónicas que generan mutaciones no sinónimas en los 10 genes inducibles por hipoxia evaluados.

- No se identificaron PNSs en las cuatro regiones exónicas de los genes estudiados que estuvieran asociados con la variación extrema de hemoglobina ni con el mal de altura en bovinos criados en la zona altoandina del Perú.

- Considerando los 10 PNSs estudiados en bovinos criados en la altura, se pudo identificar dos haplotipos propios de bovinos criollos, aunque presentes en baja frecuencia.

- No se pudo identificar un haplotipo propio de bovinos Brown Swiss con mal de altura.

\section{Agradecimientos}

Los autores desean expresar su agradecimiento a los Mg Sci Manuel More y Mayra N. Mendoza, al Consejo Nacional de Ciencia y Tecnología-CONCYTEC, al Ins- 
tituto de Investigación en Bioquímica y Biología Molecular «IIBBM-UNALM» y al Servidor HPC Bioinformática de la Dirección de Gestión de Investigación de la Universidad Nacional Agraria la Molina.

\section{Literatura Citada}

1. Accinelli RA, Lüpez LM. 2018. Enfermedades por exposición a la altura. Arch Bronconeumol 54: 115-116. doi: 10.1016/ j.arbres.2017.06.002

2. Barret JC, Fry B, Maller J, Daly MJ. 2005. Haploview: analysis and visualization of LD and haplotype maps. Bioinformatics. 21:263-265. doi: 10.1093/ bioinformatics/bth 457

3. BCF Tools. 2019. Broad Institute. [Internet]. Available in: http://samtools.github.io/bcftools/

4. Claxton JR, Ortiz P. 1996. Haematological parameters in Brown Swiss and Holstein cattle at high altitude. Trop Anim Health Pro 28: 112-116. doi: 10.1007/ BF02250734

5. Das A, Panitz F, Gregersen VR, Bendixen C, Holm L. 2015. Deep sequencing of Danish Holstein dairy cattle for variant detection and insight into potential lossof-function variants in protein coding genes. BMC Genomics 16: 1043. doi: 10.1186/s12864-015-2249-y

6. De Leon C, Manrique C, Martinez $R$, Rocha JF. 2019. Genomic association study for adaptability traits in four Colombian cattle breeds. Genet Mol Res 18: gmr18373. doi: 10.4238/gmr18373

7. Durmowicz AG, Hofmeister S, Kadyraliev TK, Aldashev AA, Stenmark KR. 1993. Functional and structural adaptation of the Yak pulmonary circulation to residence at high altitude. J Appl Physiol 74: 2276-2285. doi: 10.1152/jappl.1993.74.5.2276

8. Graham AM, McCracken KG 2018. Convergent evolution on the hypoxiainducible factor (HIF) pathway genes EGLN1 and EPAS1 in high-altitude ducks. Heredity 122: 819-832. doi: 10.1038/s41437-018-0173-z
9. Hall TA. 1999. BioEdit: a user-friendly biological sequence alignment editor and analysis program for Windows 95/98/NT. Nucl Acid S 41: 95-98.

10. Hayashi M, Sakata M, Takeda T, Yamamoto T, Okamoto Y, Sawada K, Kimura A, et al. 2004. Induction of glucose transporter 1 expression through hypoxia-inducible factor 1á under hypoxic conditions in trophoblast-derived cells. J Endocrinol 183: 145-154. doi: 10.1677/joe.1.05599

11. Holt TN, Callan RJ. 2007. Pulmonary arterial pressure testing for high mountain disease in cattle. Vet Clin N Am-Food A 23: 575-596. doi: 10.1016/j.cvfa.2007.08.001

12. Gilbert-Kawai ET, Milledge JS, Grocott MPW, Martin DS. 2014. King of the mountains: Tibetan and Sherpa physiological adaptations for life at high altitude. Physiology 29: 388-402. doi: 10.1152/physiol.00018.2014

13. Hara S, Kobayashi C, Imura N. 1999. Nuclear localization of HypoxiaInducible Factor-2á in bovine arterial endothelial cells. Mol Cell Biol Res Commun 2: 119-123. doi: 10.1006/ mcbr. 1999.0160

14. Heaton MP, Smith TPL, Carnahan JK, Basnayake V, Qiu J, Simpson B, Kalbfleisch TS. 2016. Using diverse U.S. beef cattle genomes to identify missense mutations in EPASl, a gene associated with pulmonary hypertension. F1000Research 5: 2003. 1-23. doi: 10.12688/f1000research.9254.2

15. Li H, Durbin R. 2010. Fast and accurate short read alignment with BurrowsWheeler transform. Bioinfor-matics 26: 589-595. doi: 10.1093/bioinformatics/ btp698

16. Li H, Handsaker B, Wysoker A, Fennell T, Ruan J, Homer N, 1000 Genome Project Data Processing Subgroup. 2009. The sequence alignment/ map format and SAMtools. Bioinformatics. 25: 2078-2079. doi: 10.1093/ bioinformatics/btp352 
17. Liu X, Zhang Y, Li Y, Pan J, Wang D, Chen W, Zheng $Z$, et al.. 2019. EPASI gain-of-function mutation contributes to high-altitude adaptation in Tibetan horses. Mol Biol Evol 36: 2591-2603. doi: 10.1093/ molbev/msz158

18. Martinez R, Gómez Y, Rocha JFM. 2014. Genome-wide association study on growth traits in Colombian creole breeds and crossbreeds with Zebu cattle. Genet Mol Res 13: 6420-6432. doi: 10.4238/2014.August. 25.5

19. Newman JH, Holt TN, Hedges LK, Womack B, Memon SS, Willers ED, Wheeler L, et al. 2011. High-altitude pulmonary hypertension in cattle (brisket disease): candidate genes and gene expression profiling of peripheral blood mononuclear cells. Pulm Circ 1: 462-469. doi: 10.4103/2045-8932.93545

20. Newman JH, Holt TN, Cogan JD, Womack B, Phillips III Ja, Li C, et al. 2015. Increased prevalence of EPAS1 variant in cattle with high-altitude pulmonary hypertension. Nat Commun 6: 6863. doi: $10.1038 /$ ncomms 7863

21. Ocampo NR. 2004. Determinación de valores hematológicos en bovinos Jersey tratados con ketoprofeno y sometidos a condiciones de hipoxia crónica. Tesis de Médico Veterinario. Lima. Univ. Nacional Mayor de San Marcos. 61p.

22. Picard Toolkit. 2019. Broad Institute. [Internet]. Available in: http://broadinstitute.github.io/picard/

23. Shao Y, Wellman TL, Lounsbury KM, Zhao F. 2014. Differential regulation of GLUT1 and GLUT8 expression by hypoxia in mammary epithelial cells. Am J Physiol Regul Integr Comp Physiol 307: R237-R247. doi: 10.1152/ajpregu.00093.2014

24. Semenza GL. 2005. Pulmonary vascular responses to chronic hypoxia mediated by hypoxia-inducible factor 1 . Proc Am Thorac Soc 2: 68-70. doi: 10.1513/ pats.200404-029MS
25. Simonson TS, Yang Y, Huff CD, Yun H, Qin G, Witherspoon DJ, Bai Z, et al. 2010. Genetic evidence for high altitude adaptation in Tibet. Science 329: 72-75. doi: 10.1126/science. 1189406

26. Sokal RR, Rohlf FJ. 1995. Biometry. $3^{\text {rd }}$ ed. New York: Freeman and Co. 887 p.

27. Stothard P, Liao X, Arantes AS, De Pauw M, Coros C, Plastow GS, Sargolzaei M, et al. 2015. A large and diverse collection of bovine genome sequences from the Canadian Cattle Genome Project. Gigascience 4: 49. doi: 10.1186/s13742-015-0090-5

28. Verma P, Sharma A, Sodhi M, Thakur K, Bharti VK, Kumar P, Giri A, et al. 2018. Overexpression of genes associated with hypoxia in cattle adapted to Trans Himalayan region of Ladakh. Cell Biol Int 42: 1141-1148. doi: 10.1002/ cbin. 10981

29. Vogel J, Kiessling I, Heinicke K, Stallmach T, Ossent P, Vogel P, Aulmann M, et al. 2003. Transgenic mice overexpressing erythropoietin adapt to excessive erythrocytosis by regulating blood viscosity. Blood 102: 2278-2284. doi: 10.1182/blood-2003-01-0283

30. Wang GD, Fan RX, Zhai W, Liu F, Wang L, Zhong L, Wu H, et al. 2014. Genetic convergence in the adaptation of dogs and humans to the high-altitude environment of the Tibetan plateau. Genome Biol Evol 6: 2122-2128. DOI: 10.1093/gbe/evu162

31. Wei C, Wang H, Liu G, Zhao F, Kijas $J W, M a Y, L u J$, et al. 2016. Genomewide analysis reveals adaptation to high altitudes in Tibetan sheep. Sci Rep 6: 26770. doi: 10.1038/srep26770

32. Wuletaw Z, Wurzinger M, Holt T, Dessie T, Sölkner J. 2011. Assessment of physiological adaptation of indigenous and crossbred cattle to hypoxic environment in Ethiopia. Livest Sci 138: 96-104. doi10.1016/j.livsci.2010.12.005 
33. Zeng $X$. 2016. Angus cattle at high altitude: pulmonary arterial pressure, estimated breeding value and genomewide association study. $\mathrm{Ph} \mathrm{D}$ Thesis. USA: Colorado State University. 259 p.

34. Zmajkovic J, Lundberg P, Nienhold $R$, Torgersen ML, Sundan A, Waage $A$, Skoda RC. 2018. A gain-of-function mutation in EPO in familial erythrocytosis. New Engl J Med 378: 924-930. doi: 10.1056/NEJMoa1709064
35. Zulueta JJ, Sawhney R, Kayyali U, Fogel M, Donaldson C, Huang H, Lanzillo JJ, Hassoun PM. 2002. Modulation of inducible nitric oxide synthase by hypoxia in pulmonary artery endothelial cells. Am J Respir Cell Mol Biol 26: 2230. doi: 10.1165/ajrcmb.26.1.4510 